\title{
Rezension:
}

\section{Christoph Urwyler, Die Praxis der bedingten Entlassung aus dem} Strafvollzug

Die bedingte Entlassung stellt die letzte Stufe des Strafvollzugs dar und soll Gefangene auf ein Leben in Freiheit vorbereiten. Aus amtlichen Statistiken weiss man, dass einerseits kantonale Unterschiede in der Entlassungspraxis bestehen und andererseits die bedingten Entlassungen in den letzten Jahren rückläufig sind. Über die Hintergründe dieser Befunde und die detaillierten Prozesse bei bedingten Entlassungen ist jedoch wenig bekannt. In seiner Dissertation geht Christoph Urwyler diesen Punkten nach. Auf theoretischer Ebene arbeitet er die rechtlichen Grundlagen für die bedingte Entlassung auf, aus empirischer Sicht geht er auf Basis einer umfassenden Aktenerhebung der Frage nach, wie die Entlassungsentscheide im bestehenden weiten Ermessensspielraum zustande kommen.

I. Einleitung

II. Inhalt

Zitiervorschlag:

LORENZ BIBERSTEIN, Rezension: Christoph Urwyler, Die Praxis der bedingten Entlassung aus dem

Strafvollzug, sui generis 2020, S.347 


\section{Einleitung}

1 Die bedingte Entlassung bildet die letzte Stufe des Strafvollzugs in der Schweiz (Art. 86-89 StGB1). Mit diesem Rechtsinstitut sollen verurteilte Personen an das Leben in Freiheit herangeführt werden und soll ihnen Gelegenheit gegeben werden, sich in Freiheit zu bewähren. In der Schweiz finden sich allerdings grössere Unterschiede in der Entlassungspraxis. Dies liegt einerseits daran, dass das Gesetz den Vollzugsbehörden und Gerichten grosse Freiräume in der Ausgestaltung des Verfahrens einräumt und andererseits daran, dass die Behördenorganisation und Regelungen an sich - bedingt durch das föderale System der Schweiz - sehr heterogen ausfallen. ${ }^{2}$ Diese Unterschiede in der Entlassungspraxis bergen die Gefahr einer ungleichen Behandlung. Auf statistischer Seite manifestieren sich diese Unterschiede - soweit verlässliche Zahlen dazu überhaupt verfügbar sind - in kantonal stark unterschiedlichen Entlassungsraten. Die Häufigkeit von bedingten Entlassungen nimmt zudem für die ganze Schweiz seit Jahren ab. Die Gründe für diese kantonalen Unterschiede und die rückläufige Entwicklung sind kaum erforscht. Ebenso ist häufig unklar, welche Akteure im Entscheidungsprozess (Anstaltsleitung, Staatsanwaltschaft, Begutachtende, evtl. Bewährungshilfe) welchen Einfluss auf die Entscheide der Vollzugsbehörden ausüben sowie welche Rolle die Möglichkeit einer persönlichen Anhörung der Verurteilten spielt und wie sich Screening- und Risk-Assessment-Instrumente auswirken. Die bisherigen Resultate sind «lückenhaft und über Zeit und Raum inkonsistent» (S. 8). In der Wissenschaft wird heute die bedingte Entlassung vermehrt als probates Mittel gesehen, um die soziale und berufliche Wiedereingliederung von Strafgefangenen vorzubereiten. Aus kriminalpolitischer Sicht mutet es deshalb seltsam an, dass bei einem so wichtigen Teil des Strafvollzuges grosse kantonale Unterschiede bestehen. Mit seiner Publikation möchte Christoph Urwyler einen Beitrag dazu leisten, dieses Feld besser zu beleuchten.

\section{Inhalt}

2 Im ersten Teil widmet sich der Autor den Grundlagen der bedingten Entlassung in der Schweiz. Dabei wird der Bogen geschlagen von den Anfängen der bedingten Entlassung in England und Irland und deren Aufnahme in

1 Schweizerisches Strafgesetzbuch vom 21. Dezember 1937 (StGB; SR 311.0).

2 So ist z. B. in den meisten Kantonen für die Strafvollstreckung eine administrative Vollzugsbehörde zuständig, manchmal aber auch ein spezialisiertes Vollzugsgericht oder gar die Gesamtregierung (ANDREA BAECHTOLD / JONAS WEBER / UELI HOSTETTLER, Strafvollzug. Straf- und Massnahmenvollzug an Erwachsenen in der Schweiz, 3. Aufl., Bern 2016, II/8 N11, zit. auf S. 4). die kantonalen Strafgesetze über die Vorarbeiten für ein erstes schweizerisches Strafgesetzgesetzbuch Ende des 19. Jahrhunderts bis hin zur Totalrevision des Strafgesetzbuches 2002. Vor allem die detaillierte Aufarbeitung aller Vorarbeiten der grossen StGB-Revision von 2002 ist bemerkenswert: So wurde die Rückfallverhütung erst zu diesem Zeitpunkt in den Aufgabenkatalog des Strafvollzuges aufgenommen und die Freiheitsorientierung als Grundsatz festgeschrieben. Ebenso interessant sind die dargelegten kriminalpolitischen Diskussionen, z. B. dass die bedingte Entlassung aus «rein generalpräventiven Rücksichten» (S. 34) erst nach Verbüssung von zwei Dritteln der Strafe eingeführt wurde. Abgeschlossen wird die historische Aufarbeitung mit der nach wie vor gültigen Feststellung, dass der Strafvollzug heute «weniger auf die Fürsorge und Rehabilitation, sondern vielmehr auf die Kontrolle von Rückfallrisiken und die Sicherung vor dem Täter ausgerichtet ist» (S. 49).

Im zweiten Kapitel widmet sich Urwyler dem heutigen 3 Stand der bedingten Entlassung im Strafgesetzbuch. Er geht zuerst auf die Ratio Legis ein und illustriert die formellen Voraussetzungen der bedingten Entlassung. Auch die Legalprognose wird umfassend beleuchtet, wobei auch hier wieder der grosse Ermessensspielraum der zuständigen Behörden betont wird (im Gesetzestext fehlt ein konkreter Kriterienkatalog). Dieser Spielraum birgt gemäss Urwyler die Gefahr, «dass vergangenheitsorientierte Kriterien gegenüber solchen, die sich auf gegenwärtiges oder künftiges Verhalten beziehen, ein grösseres Gewicht erhalten, als ihnen aus empirisch-kriminologischer Perspektive eigentlich zustehen sollte» (S. 67). Auch bei der Einschätzung der Persönlichkeit sieht der Autor Probleme: «Für die grosse Mehrheit der Straftäter dürfte für die Einschätzung der Täterpersönlichkeit stattdessen das intuitive Urteil des Vollzugspersonals und der Mitglieder der Vollzugsbehörde massgebend sein, das auf wenig expliziten Alltagstheorien basiert und folglich ein relativ hohes Risiko von Fehleinschätzungen aufweist» (S.70).

Die Grundlage für die später folgende empirische Unter- 4 suchung wird gelegt, indem Urwyler für die Kantone Bern, Freiburg, Luzern und Waadt die zuständigen Behörden und die Umsetzung des Entlassungsverfahrens beschreibt: Da von gesetzlicher Seite wenig Vorgaben bestehen, findet er entsprechend grössere Unterschiede in der Organisation des Strafvollzuges. Dazu kommt, «dass auch kantonale Gesetze, Verordnungen und Reglemente den Aufbau und die Abläufe des Strafvollzugs nicht umfassend und präzise regeln, so dass weniger rechtliche als vielmehr organisationsinterne Vorgaben für das behördliche Entscheidungshandeln bestimmend zu sein scheinen» (S. 121). Er kommt zum Schluss, dass «je nach Kanton und favorisiertem Vollzugsmodell 
[...] die Vorstellung über das Rechtsinstitut der bedingten Entlassung und die richtige Verfahrenserledigung variiert» (S.123).

5 Es folgt sodann ein erster statistischer Ausblick auf die Entwicklung der bedingten Entlassung auf nationaler, Konkordats- und kantonaler Ebene, wobei sich der Autor auf die amtlichen Statistiken stützt. Dabei stellt Urwyler (trotz Schwierigkeiten, basierend auf amtlichen Statistiken ein kohärentes Bild zu zeichnen) einerseits eine allgemeine Tendenz fest, bedingte Entlassungen seltener zu gewähren, und findet andererseits unterschiedliche Entlassungszahlen zwischen den Konkordaten, welche augenscheinlich ein «Ost-West-Gefälle» (S.129) zeigen.

6 Den Abschluss des Grundlagenteils bildet ein Vergleich mit dem ausländischen Recht, wobei der Fokus auf der zuständigen Behörde und den Voraussetzungen der bedingten Entlassung liegt. Bezüglich zeitlicher Voraussetzung der bedingten Entlassung ist interessant, dass die Schweiz mit der gesetzlichen Minimalfrist der Strafverbüssung von zwei Dritteln nicht nur vergleichsweise restriktiv, sondern auch starr agiert, da die bedingte Entlassung nach Verbüssung der Hälfte der Freiheitsstrafe in der Schweizer Praxis keine Rolle spielt. Dafür sei die Schweiz mit der Voraussetzung, dass bereits das Fehlen einer negativen Individualprognose als materielle Voraussetzung für die bedingte Entlassung reicht, liberaler und «täterfreundlicher» (S. 154).

7 Im zweiten Teil widmet sich Urwyler dem empirischen Forschungsstand. Ein erster Blick gilt der Auswahl, Bewertung und Gewichtung der Prognosekriterien; dazu werden der Entscheidungskontext, die Verfahrensbeteiligung und die Stellungnahmen von Anstaltsleitung, Staatsanwaltschaft, Bewährungshilfe und Fachkommission betrachtet. Weiter wird der aktuelle Kenntnisstand zur bedingten Entlassung aufgearbeitet, wobei vor allem Verbreitung und Konzeption von Risk-Assessment-Instrumenten und die kantonale Umsetzung von elementaren Verfahrensgarantien (Verteidigung, rechtliches Gehör, Begründungspflicht) im Zentrum stehen.

8 Zuletzt werden frühere empirischer Arbeiten beleuchtet, welche sich in der Schweiz schon mit der Entlassungspraxis beschäftigt haben. Eine erste Studie aus dem Jahr $1978^{3}$ stellt eine empirische Analyse der relevanten Kriterien bei der Prüfung einer bedingten Entlassung im Kanton Graubünden dar. Zwei Studien aus der französischsprachigen Schweiz aus den Jahren $1987^{4}$

3 HANSPETER häNNI, Die Praxis der bedingten bzw. probeweisen Entlassung aus dem Straf- und Massnahmenvollzug im Kanton Graubünden, Diss. Basel 1978.

4 MICHEL GRABER, La libération conditionnelle à l'épreuve du fédéralisme, KrimBull 1987, S. 4 ff. und $1994^{5}$ ermöglichten erste kantonale Vergleiche, wobei bereits hier kantonale Unterschiede festgestellt wurden, aus methodischen Gründen aber keine klaren Schlüsse über die Gründe der Unterschiede gezogen werden konnten. Die Unterschiede könnten demzufolge sowohl auf verschiedene behördliche Entscheidungsstile als auch auf unterschiedliche Gefangenenpopulationen zurückzuführen gewesen sein. Die gefundenen kantonalen Unterschiede werden - soweit vergleichbar auch von Studien aus Deutschland und Österreich gestützt, wo analoge regionale Unterschiede gefunden wurden, welche sich kaum nur auf die unterschiedliche Gefangenenpopulation zurückführen, sondern eher unterschiedliche Handlungs- und Entscheidungsstile der Richter vermuten liessen.

Im dritten Teil werden die der Forschung zugrunde lie- 9 gende Fragestellung und Methode beleuchtet. Die Arbeit soll «die praktischen Anwendungsregeln [...] der bedingten Entlassung offenlegen» (S. 184). Dazu gehören die folgenden drei thematischen Schwerpunkte: Die Häufigkeit und der Zeitpunkt der bedingten Entlassung, das Verfahren und die Ausgestaltung der bedingten Entlassung und der Faktoren, welche die bedingte Entlassung beeinflussen. Der empirische Forschungsteil besteht aus der Untersuchung der Anwendung der bedingten Entlassung in den Kantonen Waadt, Freiburg, Bern und Luzern, wobei diese vier Kantone ausgewählt wurden, da sie sich «hinsichtlich Sprachregion, Behördenorganisation und Häufigkeit der bedingten Entlassung voneinander unterscheiden» (S. 200) und der Autor davon ausgeht, dass sich die deutsch-und französischsprachigen Landesteile der Schweiz bezüglich Vollzugskultur unterscheiden. Ebenso sind so verschiedene Strafvollzugskonkordate berücksichtigt und die beiden wichtigsten Behördenmodelle vertreten (Gerichts- und Verwaltungsmodell). Um zudem eine Aussage über die zeitliche Entwicklung machen zu können, werden sowohl Akten aus dem Jahr 2010 als auch 2015 untersucht. Die benötigten Informationen werden über eine Analyse von 943 Vollzugsakten erhoben (z. B. Stammblatt der Verurteilten, Strafregisterauszug, Anstaltsbericht, Verfügung über die Straflockerung, Beschlüsse der Beschwerdeinstanz und Verfügung über die bedingte Entlassung) und mittels bivariater Analyse und logistischer Regression ausgewertet.

Zum Abschluss des dritten Teils wird ein kurzer Blick auf 10 das Gefangenenprofil der untersuchten Kantone geworfen. Dabei zeigt sich, dass sich die Gefangenenpopulationen vor allem bezüglich des Ausländeranteils relativ stark unterscheiden. So liegt der Anteil an Schweizern

5 ROBERT ROTH / NOËLLE LANGUIN / MIRANDA LINIGER / BRIGITTE MONTI / MASSIMO SARDI / FRANÇOIS-ROGER STRASSER (Hrsg.), La libération conditionnelle: risque ou chance? La pratique en 1990 dans les cantons romands, Basel 1994. 
unter den Strafgefangenen im Jahr 2015 z. B. in Waadt bei $9 \%$, in Bern bei $35 \%$. Zudem finden sich auch Unterschiede bei den Einweisungsdelikten: Während in den Kantonen Freiburg und Waadt Gewaltdelikte und Verstösse gegen das AIG $^{6}$ in der Mehrheit sind, überwiegen im Kanton Bern Widerhandlungen gegen das BetmG ${ }^{7}$.

11 Der vierte Teil widmet sich der Ergebnisse der empirischen Untersuchung. Ein erster Befund ist, dass die Häufigkeit bedingter Entlassungen in den Kantonen Luzern, Bern und Freiburg von 2010 zu 2015 zurückgegangen, im Kanton Waadt jedoch gestiegen ist. Insgesamt haben sich die kantonalen Unterschiede in der Gewährungspraxis jedoch verringert. Gleichzeitig hat sich aber der Anteil von Entlassungen mit suspensiver Bedingung von $15 \%$ auf $30 \%$ verdoppelt. Diese Bedingungen betreffen zu $90 \%$ ausländische Staatsangehörige mit einer rechtskräftigen Wegweisungsverfügung («Herr Y wird bedingt aus dem Strafvollzug entlassen, unter der Voraussetzung, dass er an diesem Tag die Schweiz verlässt», S. 230). Eine Betrachtung der Häufigkeits- und Zeitkomponente zeigt, dass Gefangene im Kanton Luzern tendenziell häufiger und früher aus dem Vollzug austreten, im Kanton Waadt seltener und später. Die Kantone Bern und Freiburg fallen zwischen diese beiden Pole.

12 Die Untersuchung der Verfahren zeigt weiter, dass die Anstaltsleitung praktisch durchgehend und in allen Kantonen am Verfahren beteiligt ist (98\% aller untersuchten Fälle). Grössere Unterschiede finden sich dagegen z. B. bei der Fachkommission, welche im Kanton Bern in nur 1\% der Fälle hinzugezogen wird, im Kanton Freiburg dagegen in $33 \%$ der Fälle. ${ }^{8}$

13 Auch bezüglich der verwendeten Instrumente zur Gefährlichkeitseinschätzung von Strafgefangenen finden sich kantonale Unterschiede. Hierbei muss zuerst unterschieden werden zwischen sogenannten «Screening-Instrumenten», welche vor der Einweisung in eine Vollzugseinrichtung zur Vollzugsplanung eingesetzt werden und «Risk-Assessment-Instrumenten», welche benutzt werden, um potenziell gefährliche Straftäter einer vertieften Rückfallrisikoabklärung zu unterziehen. Nur die Kantone Bern und Luzern setzen Screening-Instrumente zur Vollzugsplanung ein, Risk-Assessment-Instrumente werden dagegen von allen untersuchten Kantonen benutzt. Allerdings wurden zwischen 2010 und 2015 in Luzern $24 \%$ der Fälle mittels Screening-Instrument

6 Bundesgesetz über die Ausländerinnen und Ausländer und über die Integration vom 16. Dezember 2005 (AIG; SR142.20).

7 Bundesgesetz über die Betäubungsmittel und die psychotropen Stoffe vom 3. Oktober 1951 (BetmG; SR 812.121).

8 Dies ist der Sonderregelung im Kanton Freiburg geschuldet, wo bei Freiheitsstrafen ab zwei Jahren eine Stellungnahme der entsprechenden Fachkommission obligatorisch ist. überprüft, in Bern nur 6\%. Dies liegt an unterschiedlichen Regelungen, wann ein Screening-Instrument eingesetzt werden soll: In Luzern werden mit Ausnahme des Vollzugs von Geldstrafen, Bussen, Ersatzfreiheitsstrafen oder Freiheitsstrafen unter sechs Monaten alle Fälle untersucht; in Bern nur schwere Fälle mit einem Einweisungsdelikt gem. Art. 64 StGB und einer Strafdauer von über zwölf Monaten (S. 259). Obwohl diese Instrumente eher als Hilfsmittel und nicht als alleinige Entscheidungsgrundlage verwendet werden, schafft deren unterschiedlicher kantonaler Einsatz eine Ungleichbehandlung der Strafgefangenen, was einem einheitlichen Sanktionenvollzug widerspricht.

Im Rahmen der Untersuchung der Einhaltung der Ver- 14 fahrensgarantien findet der Autor erhebliche Differenzen bei der Gewährung einer persönlichen Anhörung, was auf unterschiedliche Arbeitsprozesse zurückführen ist: Während in Bern und Luzern den Gefangenen in einer schriftlichen Mitteilung der voraussichtliche Entlassungsentscheid mitgeteilt wird und gleichzeitig auch die Möglichkeit einer persönlichen Anhörung angeboten wird, werden in Freiburg und Waadt alle Verurteilten zu einer Anhörung eingeladen und wird auf die Mitteilung eines provisorischen Entscheids verzichtet. Dazu begründen diese beiden Kantone ihre Entscheide jeweils umfassend und präzise, während Bern und Luzern bei einem positiven Entscheid häufig auf eine Begründung verzichten (auch wenn belastende Massnahmen vorgesehen sind).

Bezüglich Rechtsmittel findet Urwyler weitere Unter- 15 schiede: Die höhere Beschwerdequote im Kanton Waadt lässt sich wahrscheinlich darauf zurückzuführen, dass dort Verurteilte mit einer Freiheitsstrafe von zwölf Monaten oder mehr eine amtliche Verteidigung bestellt bekommen. In Bern, Freiburg und Luzern müssen Beschwerden an die Verwaltungsbehörde gerichtet werden (und gelangen in einem allfälligen zweiten Schritt an ein Gericht), während in Waadt direkt ein Gericht zuständig ist. Allerdings ist hervorzuheben, dass die unterschiedlichen Systeme keinen Einfluss auf die Ablehnungsquote von Beschwerden haben; diese fällt mit durchschnittlich $83 \%$ für die Kantone Bern, Freiburg und Luzern (Beschwerde zuerst an die Verwaltungsbehörde) und 86\% für den Kanton Waadt (Beschwerde direkt an das Gericht) in allen untersuchten Kantonen ähnlich hoch aus.

Anhand logistischer Analysen ist es Urwyler möglich, 16 die Determinanten der bedingten Entlassungen statistisch zu überprüfen. Dabei stellt er fest, dass die Staatsangehörigkeit den grössten Einfluss auf den Gewährungsentscheid ausübt. Ausländer mit rechtskräftigem Wegweisungsentscheid, bei dem die Behörden davon ausgehen, dass sie die Schweiz verlassen werden, haben 
demzufolge die beste Chance auf eine bedingte Entlassung. Schweizer und Ausländer mit legalem Aufenthaltsstatus haben dagegen eine rund 3-mal geringere Chance auf bedingte Entlassung, Ausländer ohne Bleiberecht, bei denen die Behörden nicht davon ausgehen, dass sie die Schweiz verlassen werden, haben sogar eine 31-mal geringere Chance. Ebenfalls negativ wirken sich frühere Freiheitsstrafen aus, allerdings nicht im gleichen Ausmass wie der aufenthaltsrechtliche Status. Ein wiederholter Widerruf einer bedingten Entlassung reduziert die Chance auf eine bedingte Entlassung um das 18-Fache. Ebenfalls eine Rolle spielt der Kanton: In Bern hat ein Gefangener eine 4-mal geringere Entlassungschance als in Luzern, in Freiburg eine 7-mal geringere und in Waadt sogar eine 9-mal geringere.

17 Urwyler kommt zwar zum Schluss, dass nicht nur, wie anfangs vermutet, die Gefangenenpopulation eine Rolle bei der Entlassungswahrscheinlichkeit spielt, sondern sehr wohl auch unterschiedliche behördliche Entlassungsstile bestehen: «Den Kanton Luzern kennzeichnet ein besonders liberaler Umgang mit der bedingten Entlassung, während die Berner und Freiburger Vollzugsbehörden zu einer relativ moderaten Anwendung neigen» (S. 293). Der Kanton Waadt stellt dagegen höhere Anforderungen an die Legalprognose, hat diesen vorsichtigen Massstab von 2010 auf 2015 jedoch etwas gelockert.

18 Der fünfteund letzte Teil widmet sich der Diskussion der Befunde und den kriminalpolitischen Folgerungen. Dabei analysiert Urwyler, ob die Anwendung der bedingten Entlassung in den untersuchten Kantonen von den Grundlagen und Grundsätzen in Lehre und Rechtsprechung abweicht. Als erstes widmet er sich der Häufigkeit und dem Zeitpunkt der bedingten Entlassung: Den Rückgang der bedingten Entlassungen, zusammen mit dem Anstieg von suspensiven Bedingungen, wertet er als Hinweis auf eine restriktivere, vorsichtigere Entlassungspraxis. Interessant ist, dass in Waadt im Vergleich zu den anderen untersuchten Kantonen eine gegenläufige Entwicklung feststellbar ist: Das dortige Vollzugsgericht entscheidet häufiger zugunsten einer bedingten Entlassung mit suspensiven Bedingungen als für eine strikte Verweigerung. Dies wertet Urwyler als Indiz für ein gewandeltes Verständnis der bedingten Entlassung. So werde diese häufiger als Druckmittel verwendet, um eine rechtskräftige Wegweisung bzw. Ausschaffung vollstrecken zu können.

Die Untersuchung der sozialen Relationen zwischen der Vollzugsbehörde und den am Verfahren beteiligten Akteuren fördert unter anderem zutage, dass das Vollzugsgericht im Gegensatz zu den Vollzugsbehörden häufiger auf interdisziplinäre Expertise angewiesen ist, da es im Gegensatz zu den Vollzugsbehörden - weniger über kriminologische Spezialisten verfügt. Die wertvollste Expertise für den Gefangenen ist dabei eine positive Empfehlung der Fachkommission, gefolgt von den Gutachtenden und der Staatsanwaltschaft, wobei zwischen Anstaltsleitung, Fachkommission und Behörde grossmehrheitlich (in $82 \%$ der Fälle) ein Konsens besteht. Das Problem liegt auch hier in den kantonalen Unterschieden: «Die Tatsache, dass je nach Kanton die Beteiligung, Stellungnahme und Einflussstärke der Anstaltsleitung divergiert, führt zu einer ungleichen Behandlung gleichartiger Sachverhalte und mithin ungleichen Entlassungschancen der verurteilten Personen» (S. 317).

Bezüglich der Anwendung der persönlichen Anhörung 20 findet Urwyler klare Worte: «Der Anspruch auf rechtliches Gehör ist ein zentrales Verfahrensgrundrecht, das in allen Kantonen gilt. Deshalb ist aus grundrechtlicher Sicht die in Bern und Luzern verbreitete Praxis zu kritisieren, bei Gewährung der bedingten Entlassung mit belastenden Massnahmen oftmals auf eine persönliche Anhörung zu verzichten und selbst bei einer Verweigerung in manchen Fällen davon abzusehen» (S. 322). Ebenso kritisch äussert er sich zur unterschiedlichen Praxis der Entscheidbegründung. Speziell heikel mute das Vorgehen im Kanton Bern an, jeden fünften Negativentscheid mittels vorgefertigter Textbausteine zu begründen. Beide Vorgehensweisen führen zu Schwierigkeiten bezüglich Transparenz und Nachprüfbarkeit von Entscheidungen. Positiver sieht es bei der Rechtsweggarantie aus, wo zwar keine kantonalen Unterschiede in der Erfolgsaussicht bei einer Beschwerde, wohl aber bei der Wahrscheinlichkeit, den Beschwerdeweg überhaupt zu beschreiten, bestehen.

Die festgestellten Unterschiede in der Entlassungswahr- 21 scheinlichkeit je nach migrationsrechtlichem Status verletzen für den Autor den verfassungsrechtlichen Anspruch auf rechtsgleiche Behandlung und das Willkürverbot. Es entspreche nicht dem primären Vollzugsziel der Resozialisierung, wenn die bedingte Entlassung Ausländern nur gewährt werde, damit sie anschliessend die Schweiz verlassen (S.332). Weiter zeige die Tendenz, dass bei Sexualdelinquenten die Risiken einer bedingten Entlassung eher gescheut werden als bei anderen Gefangenen, dass - besonders bei Gewaltdelikten - anstelle rationaler Lösungen zum Nachteil der Gefangenen Emotionen Einfluss auf die Entscheidung bekommen (S.334).

Da für alle Vollzugsbehörden in den Kantonen die glei- 22 chen Entscheidungskriterien gelten, folgert Urwyler, dass die festgestellten Differenzen auf systematische Unterschiede in der Anwendung der Kriterien zurückzuführen sind, auf eine «gleiche Sachverhalte ungleich behandelnde Praxis» (S. 342). Der Grund für diese Differenzen liegt in der unterschiedlichen Vorstellung des 
Zwecks der bedingten Entlassung: Während in Luzern und Bern die bedingte Entlassung als letzte Stufe des Vollzugssystems gesehen wird, welche primär auf die Wiedereingliederung der Strafgefangenen ausgerichtet ist, wird in Freiburg und Waadt die Sicherheit der Bevölkerung neben oder gar vor die Resozialisierung des Verurteilten gestellt.

Aus diesen Befunden formuliert Urwyler entsprechende kriminalpolitische Folgerungen. Naheliegend ist, dass eine Harmonisierung der Entscheidungsprozesse nötig ist. Dies könne entweder geschehen mittels einer konkordatsübergreifenden Diskussion, um entsprechende Richtlinien zur Harmonisierung der Verfahrenspraxis zu entwickeln, oder mittels der Schaffung eines Bundesgesetzes für den Strafvollzug, welche Urwyler als wirksamer einschätzt. Weiter fordert der Autor einen verstärkten Miteinbezug der Bewährungshilfe, die damit die Perspektive eines Akteurs einbringen würde, «der für die Einschätzung der vermutlichen Auswirkungen künftiger Massnahmen über besonders geeignete Fachkompetenz verfügt» (S. 351). Speziell ausgerichtet auf den Umgang mit Ausländern ohne Bleiberecht ist die Forderung, diesen häufiger eine bedingte Entlassung zu gewähren, unabhängig von der Erwartung, ob sie die Schweiz verlassen würden oder nicht. Dafür sprächen zudem auch vollzugsökonomische Argumente: Würden diese früher bedingt entlassen, könnte der Überbelegungsproblematik, welche nicht nur hohe Kosten verursacht, «sondern die Wirkungsweise des Strafvollzugs negativ beeinflusst» (S.362), begegnet werden.

zugsstatistik. Hier schlägt er eine national einheitlich geführte Vollzugsstatistik vor, die Informationen enthält zum Anteil der für eine bedingte Entlassung infrage kommenden Personen. Weiter müsste eine solche Statistik Informationen enthalten zur Dauer der Freiheitsstrafe, zum Anlassdelikt, zum Zeitpunkt der bedingten Entlassung, zur Anzahl der Widerrufe und Rückversetzungen in den Vollzug, sowie soziodemografische Merkmale und kriminelle Vorgeschichte. Dazu benötige es die Bereitschaft, neue Methoden der Qualitätskontrolle anzuwenden, welche jedoch durch die Vereinheitlichung der kantonalen Praxis zur Rechtsgleichheit und -sicherheit beitragen würde.

Als Grund für die rückläufige Tendenz der bedingten Entlassung sieht Urwyler schliesslich die «in den letzten Dekaden enorm gestiegenen Sicherheitserwartungen an den Strafvollzug, die bei den Entscheidenden vermutlich zu einer erheblichen Verunsicherung geführt haben» (S. 366) und «die damit verbundene Angst vor politischen und medialen Reaktionen nach Vollzugsentscheiden, die sich nachträglich als Fehleinschätzungen herausstellen» (S. 367). Um dem entgegenzuwirken, brauche es eine bessere Aufklärung der Politik und des Strafvollzugs sowie der Straffälligen und der Opferhilfe über die Vorteile der bedingten Entlassung, die empirisch genügend belegt seien.

\section{Würdigung}

Mit seiner Kombination der Aufarbeitung der juristi- 26 schen Grundlage und empirischer Datenerhebung schlägt der Autor eine wertvolle Brücke zwischen zwei Fachgebieten, die sich zwar nahestehen, faktisch aber zu häufiggetrennt bleiben, und zeigt damit den Wert der empirischen Rechtsforschung. Damit ist die Arbeit sowohl für Juristen als auch für Sozialwissenschaftler interessant und gleichzeitig für Theoretiker und Praktiker gleichermassen relevant.

Die interessante Fragestellung wird methodisch sorgfäl- 27 tig und umfassend aufgearbeitet und beantwortet. Die empirische Datenerhebung bietet einen wichtigen Mehrwert zu den amtlichen Statistiken, welche bis jetzt zur Thematik der bedingten Entlassungen aus dem Strafvollzug nur eingeschränkte Aussagen ermöglichten. Die umfassende Aktenanalyse, welche mit einem grossen Erhebungsaufwand verbunden war, liefert eine grosse Masse an Zusatzinformationen und ermöglicht somit eine interessante statistische Auswertung.

Zu kritisieren bleiben nur wenige Punkte: Die umfassen- 28 de Aufarbeitung könnte gleichzeitig auch ihre Schwäche sein; um Praktiker eher anzusprechen, hätte ihr vielleicht etwas mehr Kürze gutgetan. Und für eine bessere Nachvollziehbarkeit wäre es wünschenswert, die deskriptiven Tabellen würden nicht nur Prozentangaben, sondern konsequent auch absolute Zahlen enthalten.

Der Hauptbefund der ungleichen Entlassungswahr- 29 scheinlichkeit in den verschiedenen Kantonen sollte zu denken geben, wobei Urwyler mit konkreten Gesetzesvorschlägen gleichzeitig Ansatzpunkte für eine praktische Korrektur der hier gezeigten Probleme bietet. Es ist zu hoffen, dass diese Arbeit trotz ihres Umfanges bei den zuständigen Stellen gelesen und zur Kenntnis genommen wird.

Rezensiertes Werk:

«Die Praxis der bedingten Entlassung aus dem Strafvollzug", Christoph Urwyler, Carl Grossmann Verlag, Berlin/Bern 2020, ISBN: 978-3-941159-36-5 (gedruckte Ausgabe, Hardcover), ISBN: 978-3-941159-37-2 (e-Book, Open Access) 